\title{
GOTTFRIED BENN UND DIE DOPPELTE STAATSGRÜNDUNG 1949 - ZU AUSGEWÄHLTEN PRÄMISSEN SEINES SCHWIERIGEN VERHÄLTNISSES ZU STAAT AN SICH UND ZUM BUNDESDEUTSCHEN (TEIL-)STAAT
}

\begin{abstract}
Gottfried Benn and the formation of two German states in 1949: selected aspects of his complex attitude to the state as such and to West Germany

The paper is mainly concerned with Gottfried Benn's complex attitude to the state and history. By means of introductory prefigurations, such as existential tensions related to the conflict between Protestant ethics and modern aesthetics, there emerges Benn's difficult and complex relation to the state as such, seen as a product of history, and to its particular examples, starting from the Second Reich until the initial phase of West Germany. Friedrich Nietzsche's philosophy, and in particular the philosophy of art, is of great importance in this context. This issue is discussed using Benn's key works such as Roman des Phänotyp or Doppelleben. Benn's literary and life self-creations played a vital role in his relations with the political reality and the state, which is discussed at the end of this analysis. His ambivalent relation to early West Germany has a strong biographical basis, i.e. his involvement with the history of the Nazi Germany on the one hand, and on the other - the period of his literary fame at the end of his life.

KEY WORDS: History of Germany, issues of the nation and of the state, Protestant ethics, expressionism, historiosophy, Nietzsche's philosophy, the Nazi Germany, West Germany
\end{abstract}

\section{Präfigurationen: Gottfried Benns früher Mikrokosmos zwischen protestantischer Ethik und moderner Ästhetik. Der Pastorensohn im Labyrinth der Moderne}

Gottfried Benn - Jahrgang 1886 - gehörte zu der Generation, die im späten Kaiserreich sozialisiert wurde, was nicht folgenlos für die Gestaltung seines Geistes bleiben sollte. Der Pastorensohn Benn erhielt auch im Bereich der familiären Erziehung ethische Grundlagen, die übrigens später zum Gegenstand seiner Auseinandersetzung mit der alten Glaubenswelt werden sollten. Seine Identität war allerdings 
von Anfang an durch den protestantischen Geist des Pastorenhauses vorgeprägt. Die autoritäre Figur des Vaters machte ihm jedoch die Welt der christlich-protestantischen Werte hoch problematisch: Seine Dilemmas wurden im Laufe der Zeit immer beträchtlicher. Er musste den Untergang der alten Welt der Vormoderne intensiv durchleben. Seine konfessionelle Skepsis wuchs auch durch seine Jugendlektüren, unter denen die Schriften Friedrich Nietzsches eine besondere Rolle spielten. Die Krise der protestantischen Ethik löste bei dem jungen Benn große Orientierungsdefizite aus, die dann zum ästhetisch fundierten Ausgleich im deutschen Expressionismus führen sollten. Silvio Vietta (1990) diagnostiziert diese Lage wie folgt:

Wie bereits angedeutet, läßt sich aber die expressionistische Idee des neuen Menschen und die ihr
entsprechende Rhetorik nicht isolieren von der tiefgreifenden Erfahrung der Verunsicherung, ja
Dissoziation des Ich, der Zerrissenheit der Objektwelt, der Verdinglichung und Entfremdung von
Subjekt und Objekt, Erfahrungen, die ebenfalls in dieser Radikalität literaturgeschichtlich zum
ersten Mal im Expressionismus zur Darstellung kommen. Es geht hier um das Problem der Ent-
fremdung im modernen, arbeitsteiligen, nur noch der Kategorie der Produktivität verpflichteten
Produktionsprozeß, um Verabsolutierung des Machtprinzips in der modernen Gesellschaft, um den
durch die neuen Massenmedien bedingten Strukturwandel der Öffentlichkeit, um das Problem der
Zersetzung der traditionellen Leitbegriffe der abendländischen Metaphysik, (...), die Entfremdung
von Subjekt und Objekt in der modernen Wissenschaft und Theoriebildung, (...), und die unschein-
bare, darum nicht minder tiefgreifende wahrnehmungspsychologische Umschichtung, die durch die
veränderten Wahrnehmungsbedingungen der modernen Großstadt ausgelöst wird. (S. 21)

Benn vermochte seine Orientierungskrise nur teilweise durch den deutschen Expressionismus zu kompensieren. Sein Mikrokosmos blieb durch Prämissen (vor)bestimmt, die ihre Wurzeln in der ontologisch fundierten Spannung seiner Sozialisierungsmuster hatten. Sein geistig-intellektueller Vater Friedrich Nietzsche war ein wesentlicher Gegenpol zu seiner christlich-protestantischen Erziehung, was wiederum permanente Risse in seinen lyrischen und prosaischen Texten hinterließ. Die grundsätzlich bedeutende Spannung, die durch das Gesamtwerk Benns geht, resultiert aus der frühen Präfiguration, die zwar später unterschiedlichen Verwandlungen unterlag, deren Wesen aber im Prinzip immer ein und dasselbe blieb: es waren nämlich immer wieder Versuche, den westlichen Nihilismus ästhetisch zu überwinden. Walter Lennig, der Benn-Biograph, verweist auch eindeutig auf wesentliche Einflüsse Nietzsches auf Benns kritische Wahrnehmung des westlichen Kulturkreises am Anfang des 20. Jahrhunderts:

Die Zivilisation ist kunstfeindlich, der Wohlfahrt und dem praktischen Nutzen zugetan, keine Elite kann sich sammeln und nicht das glühende Klima des Geistes. So hatte sich Nietzsche vernehmen lassen, und so jetzt, nach Kriegsende andere Autoren (Oswald Spengler vor allem), die für ihn und den Fortgang seines Geistes wichtig wurden. (Lennig, 1986, S. 66-67)

Helmut Uhlig betont dagegen die Bedeutung des Werkes Der Wille zur Macht, in dem Nietzsche diagnostiziert, die Entgötterung der Welt sei der Grund für das 
Heraufdämmern des Nihilismus im Abendland (Uhlig, 1961, S. 48-49). Benn selbst würdigt Nietzsches intellektuellen Nachlass und seine Rolle in der eigenen geistigen Entfaltung in seinem bekannten Essay Nietzsche nach 50 Jahren (1950) (Benn, 1989d, S. 495-504), der allerdings auch rezeptionskritische Elemente enthält. Diese Präfiguration brachte die für Benn spezifische Metaphysik, die ihren Ausdruck vor allem in seinem lyrischen Werk fand. Die rapide Entwicklung der akademischen Wissenschaft in der zweiten Hälfte des 19. Jahrhunderts und am Anfang des 20. Jahrhunderts brachte unter anderem - auch für Benn - neue Herausforderungen. Für ihn waren es Natur- und Geschichtswissenschaften, die seinen Auseinandersetzungen besonders relevanten Stoff lieferten. Entscheidend waren hierbei seine spätere medizinische Ausbildung und sein Kampf um die unabhängige Position des Künstlers in der modernen politischen Welt. Der geschichtliche Materialismus und die geschichtsoptimistische, teleologisch untermauerte Betrachtung des geschichtlichen Prozesses blieben für Benn Gegenstand einer permanenten Auseinandersetzung. Der Staat an sich war für Benn ein untrennbares Produkt der Geschichte. Da diese in seiner Perspektive eine ständige Bedrohung der künstlerischen Unabhängigkeit war, musste sie von ihm auch kritisch wahrgenommen werden. Bevor sein kompliziertes Verhältnis zur BRD einer kurzen Analyse unterzogen wird, ist es unumgänglich, auf dessen Vorgeschichte hinzuweisen, insbesondere auf die Bennschen intellektuell-geistigen Prämissen, die ihn auch in diesem Kontext positionieren lassen.

Der junge Benn scheint vor allem durch Nietzsches Der Wille zur Macht inspiriert worden zu sein. Dieser Text enthält einen ganzen Katalog von grundsätzlich wichtigen Fragen, die dann auch Benns existentielle Perspektiven und sein Kunstverständnis entscheidend prägten. Hier sei lediglich auf Aspekte hingewiesen, die für das Thema dieser kurzen Betrachtung von Belang sind. In dem Dreieck KunstGeschichte-Staat bewegt sich ja der intellektuelle Impetus Benns. Und das betrifft alle Textsorten, die aus seiner Feder stammten. Was zur direkten Nachfolge Nietzsches bei Benn gehört, lesen wir in dem folgenden Zitat:

Die Frage des Nihilismus ‘wozu?' geht von der bisherigen Gewöhnung aus, vermöge deren das Ziel von außen her gestellt, gegeben, gefordert schien - nämlich durch irgend eine übermenschliche Autorität. Nachdem man verlernt hat an diese zu glauben, sucht man doch nach alter Gewöhnung eine andere Autorität, welche unbedingt zu reden wüßte, Ziele und Aufgaben befehlen könnte. Die Autorität des Gewissens tritt jetzt in erster Linie (...) als Schadenersatz für eine persönliche Autorität. Oder die Autorität der Vernunft. Oder der soziale Instinkt (die Herde). Oder die Historie mit einem immanenten Geiste, welche ihr Ziel in sich hat und der man sich überlassen kann. Man möchte herumkommen um den Willen, um das Wollen eines Zieles, um das Risiko, sich selbst ein Ziel zu geben; man möchte die Verantwortung abwälzen (- man würde den Fatalismus akzeptieren). Endlich: Glück, und, mit einiger Tartüfferie, das Glück der meisten. (Nietzsche, 1980, S. 19-20)

Auch Benns Geschichtspessimismus und seine tiefe Skepsis der teleologischen Geschichtsauffassung gegenüber resultieren aus der Nietzscheanischen Inspiration. 
Es ist zugleich Benns Skepsis am Wesen der modernen Welt, deren Produkt auch der moderne Staat ist. Nietzsche schreibt dazu in Der Wille zur Macht:

Ursachen für die Heraufkunft des Pessimismus: 1. daß die mächtigsten und zukunftsvollsten Triebe des Lebens bisher verleumdet sind, so daß das Leben einen Fluch über sich hat; 2. daß die wachsende Tapferkeit und Redlichkeit und das kühnere Mißtrauen des Menschen die Unablösbarkeit dieser Instinkte vom Leben begreift und dem Leben sich entgegengewendet; 3. daß nur die Mittelmäßigsten, die jenen Konflikt gar nicht fühlen, gedeihen, die höhere Art mißrät und als Gebilde der Entartung gegen sich einnimmt, - daß, andererseits, das Mittelmäßige, sich als Ziel und Sinn gebend, indigniert (daß niemand ein Wozu? mehr beantworten kann - ); 4. daß die Verkleinerung, die Schmerzhaftigkeit, die Unruhe, die Hast, das Gewimmel beständig zunimmt, - daß die Vergegenwärtigung dieses ganzen Treibens und der sogenannten 'Zivilisation' immer leichter wird, daß der Einzelne angesichts dieser ungeheueren Maschinerie verzagt und sich unterwirft. Der moderne Pessimismus ist ein Ausdruck von der Nutzlosigkeit der modernen Welt - nicht der Welt und des Daseins. (Nietzsche, 1980, S. 27)

Diese zwei längeren Zitate waren nötig, um die bedeutendsten Prämissen zu formulieren, ohne die Gottfried Benns Kunst- und Geschichtsauffassung nicht einzusehen wäre. Dazu gehört logischerweise die Staatsproblematik. Aus diesen Zitaten wird auch ersichtlich, dass der (moderne) Staat bei Benn zur Antithese der (modernen) Kunst wurde, indem Benn in der Nachfolge Nietzsches behauptete, der moderne Staat würde zum eigentlichen Element der bürgerlichen Mediokrität. Der schöpferische Einzelne - der Künstler - sei also in Benns Perspektive an den Rand der Gesellschaft verdrängt und müsse sich demgemäß gegen ihre Trivialisierungs- und Gleichschaltungstendenzen zur Wehr setzen. Für Nietzsche und für Benn wird die Kunst angesichts der Krise der großen christlichen Kirchen des Abendlandes zur letzten denkbaren metaphysischen Tätigkeit (Nietzsche, 1980, S. 578). Wie reagiert Benn auf diese Herausforderung der Moderne? Er macht Kunst und entwickelt einen Abwehrmechanismus, den er Doppelleben nennt. Dieses Lebenskonzept sollte dann zum Gegenstand vieler Kontroversen werden.

\section{Gottfried Benns Verhältnis zu Staat an sich und zu unterschiedlichen Formen des deutschen Staates vom Kaiserreich bis zur frühen BRD. Geschichtsphilosophische und ästhetisch orientierte Konstellationen im Überblick}

Gottfried Benns Existenz (1886-1956) fiel in historisch und politisch recht abwechslungsreiche und turbulente Zeiten. Von seiner Geburt an bis zu seinem Tod änderte sich ja die Grundstruktur des deutschen Staates viermal. Seine grundsätzlich negative Einstellung zum Staat an sich und konkret zum deutschen Staat blieb konstant, mit einer einzigen Ausnahme: Es handelt sich hier um seine bekannte und ganz unerwartete Unterstützung des Dritten Reiches, die er allerdings schon im 
Jahre 1934 zurücknahm. Die Intensität seiner Negation jeden Staates wuchs deutlich nach dieser bitteren politischen Enttäuschung. Ein Sonderfall bleibt jedoch auch sein Verhältnis zur frühen BRD - in diesem Fall ist seine Einstellung weiterhin negativ, obwohl man in gewissen Aspekten von einer gewissen Ambivalenz sprechen kann. Davon wird noch die Rede sein.

Die Kunst steht bei Benn in permanenter Kollision mit (fast) jeder Staatsform und mit jeder sinnstiftenden Geschichtsauffassung. Es sind zwei entgegengesetzte Bereiche, die bei diesem Dichter das schon erwähnte Lebenskonzept Doppelleben hervorbrachten. Jeder Staatsutilitarismus und jeder Patriotismus sind ihm vollkommen fremd. Auf diesen für ihn zentralen Gegensatz kam er in vielen seinen Texten zu sprechen. Mit Wut, Ironie und Sarkasmus verteidigte er immer wieder die gesellschaftlich und sozial unabhängige Position des intellektuellen Künstlers - des poeta doctus. Charakteristisch ist in dieser Hinsicht der Rundfunkdialog Können Dichter die Welt ändern? (1930), in dem er darauf verweist, dass der (moderne) Dichter außerhalb der Geschichte und außerhalb der Staatsstrukturen stehe:

\begin{abstract}
A.: Sie haben in Ihrem Aufsatz mit dem Titel 'Zur Problematik des Dichterischen' im Aprilheft der 'Neuen Rundschau' hinsichtlich der Figur des Dichters einen Standpunkt vertreten, der ungefähr folgendes besagt: der Dichter hat keine Wirkung auf die Zeit, er greift in den Lauf der Geschichte nicht ein und kann seinem Wesen nach nicht eingreifen, er steht außerhalb der Geschichte. Ist das nicht ein etwas absoluter Standpunkt? B.: Wünschten Sie, ich hätte geschrieben, der Dichter solle sich für das Parlament interessieren, die Kommunalpolitik, die Grundstückskäufe, die notleidende Industrie oder den Aufstieg des fünften Standes? (...) A.: Sie halten also jede Beteiligung des Dichters an der Diskussion von Zeitfragen für abwegig? B.: Für Liebhaberei. Ich sehe, daß eine Gruppe von Schriftstellern für eine Abschaffung des Paragraphen 218 eintritt, eine andere für Beseitigung der Todesstrafe. Das ist der Typ von Schriftstellern, der seit der Aufklärung seine sichtbare Stellung in der Öffentlichkeit einnimmt. Sein Gebiet sind lokale Ereiferungen, freigeistige Bestrebungen, in denen der berühmte Kampf Voltaires für Calas und das j'accuse Zolas unverkennbar nachklingt. (Benn, 1989c, S. 97)
\end{abstract}

Wenn man die einzelnen Formen des deutschen Staates vom Kaiserreich bis zur BRD kurz überblickt und schließlich die Frage stellt, was konstitutiv für Benns negative Einstellung im Prinzip zu jeglicher deutschen Staatsform war, so müsste zuerst auf die ästhetisch-antibürgerliche Dimension des deutschen Expressionismus hingewiesen werden. Es fand seinen Ausdruck in dem ersten Gedichtsband Morgue und andere Gedichte (1912), in dem der Dichter sein Misstrauensvotum gegen den Geist der Belle Époque vehement und sarkastisch zur Sprache brachte. Damit war offensichtlich die schöne Fassade des deutschen Kaiserreichs mitgemeint. Benns Kritik greift aber viel tiefer - ist nicht nur Kulturkritik -, sie ist auch Kritik in der ontologischen Dimension in der Nachfolge Nietzsches. Seine frühen Gedichte aus dem Sammelband Morgue und andere Gedichte beziehen sich vor allem auf eine allumfassende Grundlagenkrise der abendländischen Zivilisation. Sie veranschaulichen auch den Nietzscheanischen ,Verlust der Mitte'. Damit wurde die Relativie- 
rungs- und die Identitätsfrage des Abendlands mit angesprochen. Auch die Weimarer Republik rief bei Benn keinen Enthusiasmus hervor. Ganz im Gegenteil: die von ihm in der neuen deutschen Staatsform diagnostizierte Mediokrität und der merkantile Geist intensivierten noch seine Entfremdungsgefühle. In seinem autobiographischen Text Neben dem Schriftstellerberuf (Kunst und Staat) aus dem Jahre 1927 schreibt er unumwunden dazu:

Hohenzollern oder Republik, das ist Jacke wie Hose. Günther, Hölderlin, Heine, Nietzsche, Kleist, Rilke oder die Lasker-Schüler - der Staat hat nie etwas für die Kunst getan. Kein Staat. Phidias starb im Kerker an Gift, sein Denunziant erhielt vom Volk Steuerfreiheit. Vergil, Dante, Petrarca, die Verbannten, unter Cäsaren oder Demokratien. Der Staat, immer bereit zu dem Geschwätz, daß die Nation sich aus inneren Kräften erneuere, hat der Kunst gegenüber keine andere Geste als die, die vom Fehlgriff lebt. (Benn, 1984d, S. 262)

Auch seine generelle Diagnose der Moderne fällt eindeutig negativ aus. Das Abendland ist aus seiner Sicht unwiderruflich zu Ende. Seine künstlerische Produktivität und ästhetische Kreativität gehören schon zur Vergangenheit. In einem anderen Text Fazit der Perspektiven (1930) lesen wir:

\footnotetext{
Immer formelhafter das Individuelle, immer genormter der Betrieb. (...) Die weiße Rasse ist zu Ende. Technische Magie, 1000 Worte Rebbach, Text genormt, Partitur aus Zahlen, das war ihr letzter Traum. Import aus Asien: Fahrräder nach Ulster, Lutscher nach Halberstadt, Bierwärmer fürs Gewerkschaftshaus. Farwell, Opportunismus von der Börse bis zur Psychiatrie! Kornloses Land, erschöpfte Schächte, leere Docks. Wer weinte um die fallenden Geschlechter - Iliaden hin und her! Das Unaufhörliche besucht den Pol, streut Erde auf Scotts Grab, bald werden die Feuerländer dort Rosen ziehen. Das Unaufhörliche, von Meer zu Meer, mondlose Welten überfrüht, hinan, hinab. (Benn, 1989b, S. 127-129)
}

Benns kurzes politisch-intellektuelles Engagement für das Dritte Reich in den Jahren 1933-34 bleibt in seiner Vita eher eine Episode. Er wollte in der Anfangsphase Hitlerdeutschlands etwas wahrnehmen, was dessen politische Realität nicht enthielt: eine rauschhafte und antidemokratische Vision Deutschlands, in der die politisch-gesellschaftliche Position des kreativen Individuums durch das Prinzip der nietzscheanischen Kunst wiederhergestellt werden sollte (Walkowiak, 2005, S. 175-202). Dies musste sich ja als pure Illusion erweisen, was er selbst in seinen Texten nach 1934 zugab. Die direkte Nachkriegszeit 1945-1949 und die frühe BRD sind Zeitspannen, in denen seine Einstellung zum Staat auf der Oberfläche seiner Selbstinszenierung weiterhin negativ ist, aber beim tieferen Hinschauen eine Ambivalenz aufweist.

Geschichtsphilosophisch und literarisch fällt Benns Roman des Phänotyp. Landsberger Fragment 1944 eine Schlüsselbedeutung zu. Der Text ist eine radikale Diagnose der modernen Wirklichkeit, und sie bezieht sich natürlich direkt auch auf die Realiät des modernen westlichen Staates. Insofern betrifft sie historisch gesehen die Weimarer Republik, aber sie antizipiert auch die Grunddimensionen der späteren 
BRD. Die BRD scheint in vielerlei Hinsicht ein Kontinuitätsgebilde der Weimarer Republik zu sein. Ihre Gründungsväter bestätigten dies ja im Jahre 1949 nicht nur im symbolischen Ausmaß.

Gottfried Benn fokussiert im Roman des Phänotyp die Grundzüge der modernen Welt, die eigentlich eine Gegenwelt seines Kunstverständnisses ist. Das Geschichtliche geht in dieser Prosa in das Übergeschichtliche über, und so ist das ein Beispiel mehr für Benns absolute Prosa. Wolfgang Bächler bestätigt diese Perspektive in seinem Aufsatz Gottfried Benn Redivivus (1950):

\footnotetext{
Auch die beiden anderen Prosastücke des Bandes Der Ptolemäer, Der Roman des Phänotyp und die Titelnovelle, nehmen das gleiche Thema zu späterer Zeit, 1944 und 1947, auf. Die Existenz des heutigen Menschen und ihre übergeschichtliche Verwurzelung wird ausgelotet, ein rein ästhetisches Weltbild als letztes Ergebnis gefestigt. 'Phänotypen' - ein aus der Erblehre entlehnter Begriff sind für Benn die Individuen, die eine Epoche innerlich repräsentieren und zum Ausdruck bringen. (Bächler, 1987, S. 210)
}

Wenn hier Der Roman des Phänotyp als generelle Zeitdiagnose mit dem geschichtsphilosophischen Hintergrund und ästhetisch orientierter Ambition betrachtet werden kann, so ist Benns autobiographischer Text mit dem markanten Titel Doppelleben ein Versuch, sich mit einigen schwierigen Schlüsselfragen auseinanderzusetzen. In dieser kurzen Analyse kann allerdings nur auf diese Aspekte hingewiesen werden, die für die Geschichts- und Staatsproblematik relevant sind. Im Weiteren werden auch einige Briefe Benns herangezogen, die in diesem Kontext bedeutungsvoll sind.

\section{Gottfried Benn nach 1945: das Konzept Doppelleben in der Realität der Besatzungszonen und in der frühen BRD. ,Tat twam asi' - , auch das bist Du' oder Selbstinszenierungen eines Monomanen}

Benns autobiographische Schrift mit dem sprechenden Titel Doppelleben, die 1949 erschien, besteht aus zwei großen Teilen: Lebensweg eines Intellektualisten (1934) und Doppelleben (1949). Der erste Teil wurde 1934 als selbständiger Text veröffentlicht und dann wieder als ein integraler Teil in Doppelleben aufgenommen. Diese beiden Texte entstanden eigentlich als Rechtfertigungsschriften unter Druck der historisch-politischen Realität: Ersterer im Dritten Reich als Antwort auf die Angriffe der NS-Diktatur, der andere als Reaktion auf Entnazifizierungsregelungen der amerikanischen Besatzungsbehörden. Benn wurde nämlich anfänglich von den Amerikanern als Nazi-Anhänger eingestuft und auf eine schwarze Liste gesetzt. Um dieses Publikationsverbot wieder aufzuheben, musste man einer entsprechenden USBehörde eine schriftliche Auseinandersetzung mit dem autobiographischen Stoff 
vorlegen, in der es erwünscht war, sich selbstkritisch auf die gegebenen Kontroversen zu beziehen.

Benn befolgte diese Anordnung, doch er nahm es eigentlich gar nicht ernst. Dieser Schritt löste aber eine beachtenswerte Resonanz aus. Es fehlte nicht an kritischen Stimmen, unter denen die von Carl Schmitt charakteristisch für Benns alte politische Allianz war. Schmitt war darüber entrüstet, dass Benn sich überhaupt auf die Erfordernisse des Entnazifizierungsverfahrens eingelassen hatte (Lethen, 2006, S. 238-244). Benn selbst schrieb aber darüber unmissverständlich in einem Brief an seinen Freund Friedrich Wilhelm Oelze am 25. 12. 1949:

Schließlich habe ich mir gesagt, man muss auch nicht zu schwierig und bedenklich in allem sein (wozu ich neige), sondern ruhig 5 gerade sein lassen, bzw. aus 5 Vier zu machen. Wer will, kann immer aus den alten Sachen mir tausend Stricke drehn und aus den mannigfachsten Stellen sich Gift für seine Pfeile holen. (Benn, 1982b, S. 279)

Damit wurde eines der wichtigsten Probleme angesprochen, das Benns Verhältnis zuerst zur Nachkriegsrealität und dann zum bundesdeutschen (Teil-)Staat profiliert: Denn die Kontroversen um seine NS-Phase hören bis heute nicht auf.

In diesem Kontext klingt die kritische Stimme Peter de Mendelssohns besonders deutlich. Seine Kritik gegen Benns Verhalten in der Nazizeit hatte auch eine beachtliche mediale Wirkung. Darauf bezieht sich Benn direkt in einem Brief an Ernst Jünger im Jahre 1952:

Wir haben einen neuen üblen Feind gemeinsam: Herr Peter de M. Er wollte neulich am Rias ein gemeines Pamphlet gegen mich starten, aber ich habe es durch Klageandrohung zu verhindern gewusst. Jetzt erscheint, wie ich höre, hier in Berlin ein Essayband von ihm mit den Machwerken gegen Sie, Heidegger, Salomon, mich u. a. Nun, wir werden es überstehn. (Benn, 2006b, S. 35)

Benn meint hier den Band von Peter de Mendelssohn mit dem Titel: Der Geist in der Despotie. Versuche über die moralischen Möglichkeiten des Intellektuellen in der totalitären Gesellschaft (1953).

Da die Besatzungsbehörden und später die BRD wenigstens formal bemüht waren, sich mit dem NS-Ballast kritisch auseinanderzusetzen, war Benns Position recht zwiespältig: einerseits wurde er wegen seines Engagements für das Dritte Reich vor allem in links-liberalen Kreisen heftig kritisiert, und andererseits begann schon im Jahre 1949 sein später literarischer Ruhm. Das beeinflusste auch seine Einstellung zu der Nachkriegsrealität und später zur BRD. Er verhalf selbst dazu, mit seinen alten und neuen Texten wieder an die literarische Öffentlichkeit treten zu dürfen: sein Doppelleben wurde positiv bewertet, und das Publikationsverbot wurde aufgehoben. Gleichzeitig setzte eine wachsende Rezeptionswelle der Bennschen Werke ein. Helmut Lethen kommentiert sarkastisch, es sei das größte Comeback seit Lazarus gewesen (Lethen, 2006, S. 261-271). Benn wurde sogar quasi zu einer Identifikationsfigur und damit zu einem Entlastungszeugen eines durchschnittlichen, in die 
schwierige deutsche Geschichte involvierten BRD-Bürgers. Dies hatte auch gravierende Folgen für die mentale Sphäre der bundesrepublikanischen Gesellschaft der späten 40er und der 50er Jahre.

Benns Verhältnis zur frühen BRD ist nicht eindeutig. Auf der Ebene der Selbstinszenierung - gern auch in seinen zahlreichen Briefen aus dieser Zeitspanne exponiert er Distanz, Skepsis und Misstrauen. Darin klingen noch deutlich seine alten Aversionen gegen die früheren deutschen Staatsformen nach, sowie seine generelle und fast programmatische Abneigung gegen jede Staatsform. Das Konzept Doppelleben war ihm dabei sehr dienlich - er betonte immer wieder das Janusgesicht des modernen Menschen, was ihm die Flucht vor potentiell möglichen neuen Attacken ermöglichte. Unbestimmtheit und Uneindeutigkeit der modernen Persönlichkeit waren für ihn Instrumente, die er sehr gerne in Doppelleben gebrauchte. Sie scheinen noch in der Nachfolge seiner expressionistischen Erfahrungen zu stehen. Dieses Misstrauen und Argwohn gegen alles Fassadenhafte hatte ja bei ihm tiefe Wurzeln. In manchen Briefen dagegen wird seine halb ausgedrückte Zufriedenheit damit deutlich, dass er im amerikanischen Sektor Berlins lebt (und dann in Westberlin). Diese Stadthälfte war formal gesehen kein integraler Teil der BRD, aber sie vertrat den Westen, und insofern ist es in diesem Kontext wichtig. In diesem Geiste schreibt Benn zum Beispiel an Ernst Jünger am 4. 07. 1950.:

Inzwischen war hier der berühmte Kongress, mit dem ich allerdings nicht das geringste zu tun hatte. Man hatte nur die Unverfrorenheit, mir für die Eröffnungsvorstellung im Titaniapalast eine Eintrittskarte: II Parkett, 12 Reihe zuzusenden - auf solche Plätze pflege ich nicht zu gehen. Immerhin war es für West Berlin ganz gut, daß sich internationale Redner gegen die Ostintellectuellen wandten, die ja äußerst aggressiv gegen jeden Einzelnen von uns immer wieder vorgehn. Alles war in Berlin versammelt: Presse, Radiostationen, Chefredaktöre, Politiker - und einige Ausländer, die mich privat besuchten, zu sprechen, war ganz anregend. (Benn, 2006a, S. 18)

Benns Briefe stellen im allgemeinen eine aufschlussreiche und interessante Quelle von Strategien der Selbstinszenierung eines Monomanen dar. Seine Briefe nach 1945 bringen dagegen einen frappanten Stoff in dieser Hinsicht - auch sein Verhältnis zur BRD wird in ihnen entsprechend stilisiert. Bevor aber die wichtigsten Prämissen in diesem Kontext kurz analysiert werden, lohnt es sich noch, die Dimension seiner literarischen Texte aus dieser Periode mit zu berücksichtigen. Sie sind nämlich ein bedeutendes ästhetisches und geschichtsphilosophisches Pendant zu seinen nicht-literarischen Texten.

Die bereits angedeutete ,tat twam asi'-Formel, die schon in Benns essayistisch fundiertem Prosatext Der Ptolemäer. Berliner Novelle, 1947 erscheint, ist für ihn eine Basis für Selbstgestaltungsstrategien und Selbstinszenierungen. Sie korrespondiert direkt mit seiner viel zitierten Überzeugung, dass es die Wirklichkeit nicht gebe und dass ergo die einheitliche Persönlichkeit nur ein Wahngebilde sei. Diese Ansicht war ein idealer Ausgangspunkt auch für das Konzept Doppelleben. In Der Ptolemäer 
kommt die schon bekannte Dichotomie Geschichte(Staat)-Kunst in einer anderen Opposition wieder vor: diesmal ist es der Gegensatz ,Verbrecher und Mönche‘. Benn schreibt dazu:

Die Lage barg noch viele Möglichkeiten und Deutungen, doch eines erschien mir evident. Das kommende Jahrhundert würde die Männerwelt in einen Zwang nehmen, vor eine Entscheidung stellen, vor der es kein Ausweichen mehr gab mit keiner Konzession, mit keinem Blinzeln, mit keinem Schwarzhandel, mit keiner Emigration - sie mußten sich entscheiden. Das kommende Jahrhundert würde nur noch zwei Typen zulassen, zwei Konstitutionen, zwei Reaktionsformen: diejenigen, die handelten und hoch wollten und diejenigen, die schweigend die Verwandlung erwarteten -: Verbrecher und Mönche, etwas anderes würde es nicht mehr geben. Die Orden, die Brüder werden vor dem Erlöschen noch einmal auferstehen. Ich sehe an Wassern und auf Bergen neue Athos und neue Monte Cassinos wachsen, - schwarze Kutten wandeln in stillem, in sich gekehrten Gang. Jenseits der Gegensätze von Erkennen und Erkanntem, außerhalb der Kette von Geburt und Wiedergeburt. Durch Einsamkeit, Riten und Verzicht auf das Gewohnte wird die autistische Realität die Weltausweitung löschen und in einem stummen gefassten tat twam asi, auch das bist Du, wird sich die Vereinigung mit der verlorenen Dingwelt vollziehn. (Benn, 1984a, S. 207)

Dieses längere Zitat veranschaulicht, wie Benns geschichtsphilosophische Selbstinszenierung aussieht. Er stilisiert sich dadurch zu einem Dichter und Weisen zugleich, der seine Distanz der geschichtlichen Welt gegenüber betont und sich vor dem Staat in eine Freiheitsnische der hohen Kunst flüchtet. Damit glaubt er, auch seine intellektuelle Unabhängigkeit und geistige Freiheit zu demonstrieren und zu schützen.

Auch in Doppelleben finden sich Passagen, die die ,tat-twam-asi'-Formel ideenmässig weiterführen. Benn greift diesmal tief in die Kulturgeschichte des Abendlandes ein, um seine Ansicht anhand der mythischen Stoffe zu begründen. In einem recht eruptiven Ton schreibt er:

Unser Kulturkreis begann mit Doppelgestalten: Sphinxen, Zentauren, hundsköpfigen Göttern und befindet sich mit uns in einer Kulmination von Doppelleben: wir denken etwas anderes als wir sind, oder, wie die Drei alten Männer es ausdrücken: Wir lebten etwas anderes als wir waren, wir schrieben etwas anderes als wir dachten, wir dachten etwas anderes als wir erwarteten und was übrig bleibt, ist etwas anderes als wir vorhatten. Die Einheit der Persönlichkeit ist eine fragwürdige Sache. (...) Ich bin also Dualist, Anti-Synthetiker oder, um nochmals die Drei alten Männer zu zitieren, ich halte vor dem Unvereinbaren, mein Streben nach Einheit beschränkt sich auf das jeweilig in meinen Händen zur Bearbeitung befindliche Blatt. (...) Heute und hier, keine Allgemeinheiten und siderischen Dränge - das ist eine gute Grundlage für Doppelleben und mein eigenes Doppelleben war mir nicht nur immer sehr angenehm, ich habe es sogar mein Leben lang bewußt kultiviert. (Benn, 1984b, S. 448-449)

Benn setzt diese Selbststilisierung in Doppelleben fort, indem er unterschiedliche Themen und Stoffe bemüht. Auch Knut Hamsuns Prosa bietet ihm eine Möglichkeit in diese Richtung. Der alte Gegensatz Kunst vs. Geschichte (Staat) wird aufs neue ausgespielt: Diesmal ist Knut Hamsuns Text Auf überwachsenen Pfaden Ausgangspunkt für Benn, der seine Tiraden gegen den generell bürgerlichen Staat weiter macht: 
Diese Haltung ist interessant [gemeint ist die Haltung des Erzählers in diesem Text - M.W.], denn sie führt vor ein entscheidendes Problem. Diese Haltung führt vor die große Trennung, die durch die abendländische Welt geht: einerseits die Kunst und alles, was mit ihr zusammengehört, und andererseits das gute, warme, pausenlose Familienleben, gestützt durch Versicherungspolicen, Renten, ,Ansprüche‘ bis zu den Lebensabenden, garantiert und wohltemperiert von einer Art Bierwärmer: dem Staat. (Benn, 1984b, S. 458-459)

Nach dieser Darstellung, in der die literarisch-existientiellen Prämissen des Bennschen Konzepts Doppelleben aus seinem gleichnamigen Text mit einem primär wichtigen Kontext aus der Zeitgeschichte - nämlich mit dem Entnazifizierungsverfahren und seinen für Benn relevanten Konsequenzen kurz analysiert wurden, ist es nun notwendig, stichpunkartig auf die anderen historisch-politischen Elemente hinzuweisen, die sein Verhältnis zur BRD mit prägten.

Der Akt der doppelten Staatsgründung selbst 1949 scheint keine bedeutende Zäsur in Benns Taktik zu bewirken. Es ist für ihn lediglich eine Art Fortsetzung der Realität der westlichen Besatzungszeit. Der bundesdeutsche (Teil-)Staat bleibt ihm in der weitergeführten Selbstinszenierung eine Staatsform mehr, die sich als eine neue Erscheinungsform des Geschichtlichen und des Bürgerlichen und damit als eine Antithese seines Kunstprinzips erweist. Dies ist aber die Dimension seiner Selbstinszenierung und somit ein Eindruck, den er erwecke wollte. Benn als ein Elfenbeinturmbewohner kreiert sich weiter als einen bewusst wirklichkeitsfernen Monomanen, aber sein Verhältnis zur BRD ist nicht so offensichtlich und eindeutig. Es ergibt sich vor allem aus seinen Briefen aus dieser Zeitspanne. Wenn man die Oberfläche dieser brieflichen Selbstgestaltung und Tarnung durchschaut, erkennt man auch, wie ambivalent sein Bezug zu dem neuen westdeutschen Staat war.

Wie schon in der oben zitierten Briefpassage (Brief an Ernst Jünger) ersichtlich wird, ist es Benn nicht ganz gleichgültig, dass er in der Realität des Kalten Krieges auf der westlichen Seite lebt. Trotz aller Kritik und allem Sarkasmus scheint er damit zufrieden zu sein, dass er nicht unter der sowjetischen Satrapie zu leben hatte. Allerdings nahm er auch wahr, dass Westberlin durch die Teilung Deutschlands viel von seiner früheren Bedeutung verloren hatte. Dies wird auch von ihm in dem Text Berlin zwischen Ost und West (1955) klar festgestellt (Benn, 1989a, S. 589-591).

Gleichzeitig aber - wie bereits erwähnt - beginnt die Phase seines späten Ruhmes. Er wird zu einem hochgeschätzten Dichter und Literaturkenner. Es beginnen sich Einladungen für ihn zu häufen: er wird auch als Literaturkritiker und Literaturexperte anerkannt. Er geht auf Reisen, um Vorträge zu halten. Friedrich Wilhelm Wodtke schreibt in seiner Biographie über eine Zäsur in dieser Hinsicht, über die erste bedeutende und öffentliche Ehrung Benns:

Das folgende Jahr sah ihn dreimal außerhalb Berlins: am 2. Mai 1951 feierte er seinen 65. Geburtstag mit Frau und Tochter, Bernard von Brentano und Frau und Karl Schwedhelm bei seinem Verleger Max Niedermayer in Wiesbaden und hielt am 21. August 1951 in der Universität Marburg auf 
Einladung des Rektors, des Romanisten Alwin Kuhn und des Germanisten Arthur Henkel vor dem Internationalen Ferienkurs seinen grundlegenden Vortrag 'Probleme der Lyrik', der auch vom Hessischen Rundfunk aufgenommen und gesendet wurde. Die erste öffentliche Ehrung Gottfried Benns folgte bald darauf mit der Verleihung des 'Georg-Büchner-Preises' durch die 'Deutsche Akademie für Sprache und Dichtung' in Darmstadt, der ihm am 21. Oktober 1951 dort in einer Feierstunde in Anwesenheit des hessischen Kultusministers Metzger von Dr. Rudolf Pechel als Präsidenten der Akademie überreicht wurde. (Wodtke, 1970, S. 81)

Benn pflegte darüber in seinen Briefen und autobiographischen Texten trocken und sachlich zu berichten, aber die BRD war ja der erste deutsche Staat, der ihn durch seine Institutionen so unmissverständlich ehrte und auszeichnete. Und dies war die positive, nicht sofort erkennbare Dimension seiner Ambivalenz. Sein Brieffreund Friedrich Wilhelm Oelze machte sogar in einem Brief an Benn eine Anspielung auf einen potentiell möglichen Nobelpreis für ihn. Benn aber erwiderte auf eine für ihn charakteristische Art und Weise:

Nobelpreis. Bitte keine Witze! Ich weiss, wo ich hingehöre u. wo nicht. Bis aufs Letzte werde ich meine Fragwürdigkeit verteidigen u. immer von Neuem unter Beweis stellen. Für die Verteilung scheint mir z.Z. Th. Mann ganz massgeblich zu sein. Alles seine Spezis: Hesse, Gide, Eliot auch. Die Preisgekrönten haben nämlich Mitbestimmungsrecht bei den neuen Rittern, werden gefragt u.s.w. (Benn, 1982a, S. 183)

Benn aber - schon seiner literarisch-intellektuellen Position nicht nur in der BRD bewusst - schreibt über den süßlich-bitteren Geschmack des großen Ruhmes in einem Brief an Hans Paeschke. Und diese Reflexion scheint keine Selbstinszenierung zu sein, obwohl sie von der alten antistaatlichen Rhetorik nicht ganz frei ist:

Der Ruhm hat keine weißen Flügel, sagt Balzac; aber wenn man wie ich die letzten 15 Jahre lang von den Nazis als Schwein, von den Kommunisten als Trottel, von den Demokraten als geistig Prostituierter, von den Emigranten als Renegat, von den Religiösen als pathologischer Nihilist öffentlich bezeichnet wird, ist man nicht so scharf darauf, wieder in diese Öffentlichkeit einzudringen. Dies um so weniger, wenn man sich dieser Öffentlichkeit innerlich nicht verbunden fühlt. (Benn, 1984c, S. 350)

Gottfried Benns Verhältnis zur BRD ist also problematisch und ambivalent. Der Akt der doppelten Staatsgründung legte ihm allerdings nahe, dass er von da an auf der westlichen Seite des geteilten Deutschlands zu leben hatte. Und er schien darin einen selten zum Ausdruck gebrachten Mehrwert zu erblicken.

\section{Resümee}

Benns Verhältnis zu Staat an sich und dann konkret zur frühen BRD ist durch Prämissen bedingt, die in dieser kurzen Betrachtung nur ansatzweise besprochen werden konnten. Zweifelsohne spielen hier die Hauptrolle solche Prämissen, wie seine soziale Herkunft, seine expressionistischen Erfahrungen und sein Reifungspro- 
zess in der labyrinthischen Realität der Moderne. Eine Bedeutung hat dabei auch die turbulente, historische Zeit, in der Benn lebte. Der Staat - unabhängig von seiner historischen Gestalt - galt ihm permanent als ein Produkt des Geschichtlichen. Der Gegensatz Kunst - Geschichte(Staat) gehörte permanent zu den Hauptvoraussetzungen seines Denkens.

Sein Verhältnis zum Staat wurde erst in der frühen BRD ambivalent (von der kontroversen NS-Phase in seinem Leben abgesehen). Zwar galt einerseits immer noch seine Skepsis und Misstrauen dem bundesdeutschen Teilstaat gegenüber weiter, aber andererseits bewirkte sein später Ruhm, dass er auch Positives wahrnahm. Dies wird deutlich, wenn man seine ironisch-sarkastische Maske durchschaut und seine Selbstinszenierung und Tarnungsstrategien richtig erkennt. Benn als ein großer Vertreter der monomanen Kunst wurde eben in der frühen BRD anerkannt, und damit wurde er zu einem berühmten Dichter, dessen Werke bis heute gelesen und rezipiert werden.

\section{Bibliographie}

Bächler, W. (1987). Gottfried Benn Redivivus (Original 1950). In B. Hillebrand (Hrsg.), Über Gottfried Benn. Kritische Stimmen 1912-1956 (S. 207-213). Frankfurt a. M.: Fischer Taschenbuch.

Benn, G. (1982a). Brief an Friedrich Wilhelm Oelze (Original 1949). In H. Steinhagen \& J. Schröder (Hrsg.), Gottfried Benn. Briefe. Briefe an F. W. Oelze 1945-1949 (Brief Nr. 398, S. 181-183). Frankfurt a. M.: Fischer Taschenbuch.

Benn, G. (1982b). Brief an Friedrich Wilhelm Oelze (Original 1949). In H. Steinhagen \& J. Schröder (Hrsg.), Gottfried Benn. Briefe. Briefe an F. W. Oelze 1945-1949 (Brief Nr. 459, S. 277-280). Frankfurt a. M.: Fischer Taschenbuch.

Benn, G. (1984a). Der Ptolemäer (Original 1947). In B. Hillebrand (Hrsg.), Gottfried Benn: Prosa und Autobiographie. In der Fassung der Erstdrucke (193-234). Frankfurt a. M.: Fischer Taschenbuch.

Benn, G. (1984b). Doppelleben (Original 1949). In B. Hillebrand (Hrsg.), Gottfried Benn: Prosa und Autobiographie. In der Fassung der Erstdrucke (S. 355-479). Frankfurt a. M.: Fischer Taschenbuch.

Benn, G. (1984c). Ein Berliner Brief (Original 1948). In B. Hillebrand (Hrsg.), Gottfried Benn: Prosa und Autobiographie. In der Fassung der Erstdrucke (S. 349-354). Frankfurt a. M.: Fischer Taschenbuch.

Benn, G. (1984d). Neben dem Schriftstellerberuf (Kunst und Staat) (Original 1927). In B. Hillebrand (Hrsg.), Gottfried Benn: Prosa und Autobiographie. In der Fassung der Erstdrucke (S. 259-267). Frankfurt a. M.: Fischer Taschenbuch.

Benn, G. (1989a). Berlin zwischen Ost und West (Original 1955). In B. Hillebrand (Hrsg.), Gottfried Benn: Essays und Reden. In der Fassung der Erstdrucke (S. 589-591). Frankfurt a. M.: Fischer Taschenbuch.

Benn, G. (1989b). Fazit der Perspektiven (Original 1930). In B. Hillebrand (Hrsg.), Gottfried Benn: Essays und Reden. In der Fassung der Erstdrucke (S. 125-129). Frankfurt a. M.: Fischer Taschenbuch.

Benn, G. (1989c). Können Dichter die Welt ändern? (Original 1930). In B. Hillebrand (Hrsg.), Gottfried Benn: Essays und Reden. In der Fassung der Erstdrucke (S. 97-103). Frankfurt a. M.: Fischer Taschenbuch. 
Benn, G. (1989d). Nietzsche nach 50 Jahren (Original 1950). In B. Hillebrand (Hrsg.), Gottfried Benn: Essays und Reden. In der Fassung der Erstdrucke (S. 495-504). Frankfurt a. M.: Fischer Taschenbuch.

Benn, G. (2006a). Brief an Ernst Jünger (Original 1950). In H. Hof (Hrsg.), Gottfried Benn - Ernst Jünger. Briefwechsel 1949-1956, (Brief Nr. 8, S. 18). Stuttgart: Klett-Cotta.

Benn, G. (2006b). Brief an Ernst Jünger (Original 1952). In H. Hof (Hrsg.), Gottfried Benn - Ernst Jünger. Briefwechsel 1949-1956 (Brief Nr. 26, S. 34-35). Stuttgart: Klett-Cotta.

Brode, H. (1978). Benn-Chronik. München: Hanser.

Lennig, W. (1986). Gottfried Benn. Reinbek bei Hamburg: Rowohlt Taschenbuch.

Lethen, H. (2006). Der Sound der Väter. Gottfried Benn und seine Zeit. Berlin: Rowohlt.

Mendelssohn, P. de (1953). Der Geist in der Despotie. Versuche über die moralischen Möglichkeiten des Intellektuellen in der totalitären Gesellschaft. Berlin: Herbig.

Nietzsche, F. (1980). Der Wille zur Macht. Stuttgart: Kröner.

Uhlig, Helmut (1961). Gottfried Benn. Berlin-Dahlem: Colloquium Verlag Otto H. Hess.

Vietta, S (1990). Probleme - Zusammenhänge - methodische Fragen. In S. Vietta \& H.-G. Kemper (Hrsg.), Expressionismus (S. 21-213). München: Fink.

Walkowiak, M. (2005). Kunst, Geschichte und der Standort des Intellektuellen. Gottfried Benn und die Kontroversen der Moderne. Frankfurt a. M.: Lang.

Wodtke, F. W. (1970). Gottfried Benn. Stuttgart: Metzler. 